\title{
ON THE TOPOLOGY OF TANGENT BUNDLES
}

\author{
L. AUSLANDER ${ }^{1}$ AND R. E. MACKENZIE
}

1. Introduction. Let $M$ be an $n$-dimensional differentiable manifold. If $m$ is a point of $M$ then denote by $T(m)$ the tangent space to $M$ at $m$. For each $m$ in $M, T(m)$ has the algebraic structure of Euclidean $n$-space $V^{n}$. That is to say, if $\left(x_{1}, x_{2}, \cdots, x_{n}\right)$ are coordinates in a coordinate neighborhood containing the point $m$ then the correspondence

$$
\left(a_{1}, a_{2}, \cdots, a_{n}\right) \rightarrow a_{1}\left(\frac{\partial}{\partial x_{1}}\right)_{m}+a_{2}\left(\frac{\partial}{\partial x_{2}}\right)_{m}+\cdots+a_{n}\left(\frac{\partial}{\partial x_{n}}\right)_{m}
$$

is a vector space isomorphism of $V^{n}$ onto $T(m)$.

The tangent bundle $\Im(M)$ of the manifold $M$ consists of the ordered pairs $(m, v)$ where $m \in M$ and $v \in T(m)$. Therefore, as a point set only, $\Im(M)$ is $M \times V^{n}$. We shall assume that the reader is familiar with the fibre space topology which is customarily assigned to $J(M)$. (For a description of this topology and the facts concerning fibre bundles which we shall need in other parts of this paper see N. Steenrod, The topology of fibre bundles, Princeton, 1951.) For many manifolds $M$ this topology differs from the topology of the product space $M \times V^{n}$. This fact indicates that it is the assignment of this topology which makes $J(M)$ an interesting mathematical object. It is the purpose of this paper to present an intrinsic characterization of this topology.

2. General considerations. Let $X$ be a vector field defined on some open subset $U$ of $M . X$ assigns to each point $m$ of $U$ a vector $X(m)$ in $T(m)$. For each point $m$ in $M$ let $F(m)$ be the vector space of all real-valued functions which are of class $C^{1}$ on some neighborhood of $m$. If $m$ is in $U$ and $f$ is in $F(m)$ then define $\langle X(m), f\rangle$ to be the value of the differential $d f$ at the vector $X(m)$.

Let $m_{0} \in U$ and $f \in F\left(m_{0}\right)$. Then there is a neighborhood $V$ of $m_{0}$ which is contained in $U$ such that $\langle X(m), f\rangle$ is defined on $V .\langle X, f\rangle$ is then a real-valued function on $V$.

Definition. The vector field $X$ is said to be continuous at the point $m_{0}$ if $\langle X, f\rangle$ is continuous on a neighborhood of $m_{0}$ for each $f$ in

Received by the editors December 1, 1958.

1 Partially supported by a National Science Foundation contract during the preparation of this paper. 
$F\left(m_{0}\right) . X$ is said to be continuous on $U$ of it is continuous at each point of $U$.

Let $\pi: \Im(M) \rightarrow M$ be the projection defined by $\pi(m, v)=m$. If $X$ is a vector field on an open subset $U$ of $M$ we may define $\chi: U \rightarrow J(M)$ by $\chi(m)=(m, X(m))$. The correspondence $\chi$ then satisfies $\pi \chi=\mathrm{iden}$ tity on $U$. The vector fields on $U$ are in one-to-one correspondence with the mappings $\chi$ of $U$ into $J(M)$ which satisfy $\pi \chi=$ identity.

The relationship between our definition of continuous vector field and the problem of characterizing the topology in $J(M)$ is indicated by the following lemma.

Lemma. A vector field $X$ defined on a coordinate neighborhood $U$ of $M$ is continuous on $U$ if and only if the associated mapping $\chi$ is continuous in the usual topology on $\mathrm{J}(M)$.

Proof. Let $\left(x_{1}, x_{2}, \cdots, x_{n}\right)$ be coordinates in $U$. Let $X=v_{1} \partial / \partial x_{1}$ $+v_{2} \partial / \partial x_{2}+\cdots+v_{n} \partial / \partial x_{n}$ where $v_{1}, v_{2}, \cdots, v_{n}$ are functions on $U$. If $m \in U$ and $f \in F(m)$ then $d f=w_{1} d x_{1}+w_{2} d x_{2}+\cdots+w_{n} d x_{n}$ where $w_{1}, w_{2}, \cdots, w_{n}$ are continuous functions on some neighborhood $V$ of $m$ which is contained in $U$. Hence $\langle X, f\rangle=v_{1} w_{1}+v_{2} w_{2}+\cdots+v_{n} w_{n}$ on $V$.

Let $p_{j}\left(x_{1}, x_{2}, \cdots, x_{n}\right)=x_{j}$ for $j=1,2, \cdots, n$. If we assume that $X$ is continuous then $v_{j}=\left\langle X, p_{j}\right\rangle$ is continuous on $U$ for $j=1,2, \cdots, n$, and so $\chi$ is continuous in the usual topology on $J(M)$. Conversely, if we assume that $\chi$ is continuous in the usual topology on $\Im(M)$ then $v_{1}, v_{2}, \cdots, v_{n}$ are continuous, and hence $\langle X, f\rangle$ is continuous on $V$. Since this is true for every $m \in U$ and $f \in F(m)$ we find that $X$ is continuous on $U$.

3. The characterization of the topology of $J(M)$. In order to distinguish the two topologies which we shall be considering on the same point set $J(M)$ we shall refer to the usual fibre bundle topology as the o-topology on $\Im(M)$. The actual topology of $J(M)$ will be called the *-topology. The five conditions which we shall assume for the *-topology are the following:

(i) $J(M)$ is a regular space.

(ii) Each point of $J(M)$ has an open neighborhood in the *-topology whose closure in the o-topology is compact in the o-topology.

(iii) The projection $\pi$ is a continuous mapping.

(iv) For each point $m$ in $M$ the topology induced on $\pi^{-1}(m)$ by the *-topology is the same as that induced by the o-topology.

(v) If $X$ is any continuous vector field on an open set $U$ in $M$ then the associated mapping $\chi$ is a continuous mapping of $U$ into $J(M)$. 
ThEOREM. If the *-topology satisfies conditions (i)-(v) then it is the same as the o-topology.

Proof. If $U$ is any coordinate neighborhood of $M$ and $\left(x_{1}, x_{2}, \cdots, x_{n}\right)$ are coordinates in $U$ then we may associate with these a one-to-one mapping $h$ of $U \times V^{n}$ onto $\pi^{-1}(U)$ defined by

$$
\begin{aligned}
h\left(m,\left(a_{1}, a_{2}, \cdots, a_{n}\right)\right) & \\
& =\left(m, a_{1}\left(\frac{\partial}{\partial x_{1}}\right)_{m}+a_{2}\left(\frac{\partial}{\partial x_{2}}\right)_{m}+\cdots+a_{n}\left(\frac{\partial}{\partial x_{n}}\right)_{m}\right) .
\end{aligned}
$$

Now suppose that under the assumptions (i)-(v) we may prove that for every coordinate neighborhood $U$ the corresponding mapping $h$ is a homeomorphism. Let $U$ and $U^{\prime}$ be two overlapping coordinate neighborhoods and let $h$ and $h^{\prime}$ be their corresponding mappings, respectively. Define $g=h^{-1} h^{\prime}$ on $\left(U \cap U^{\prime}\right) \times V^{n}$. Then $g\left(m,\left(a_{1}, a_{2}, \cdots, a_{n}\right)\right)=\left(m, l(m)\left(a_{1}, a_{2}, \cdots, a_{n}\right)\right)$ where $l(m)$ is a nonsingular linear transformation of $V^{n}$, that is, an element of $G L(n)$. The correspondence $m \rightarrow l(m)$ is a continuous mapping from $U \cap U^{\prime}$ into $G L(n)$. Consequently $\Im(M)$ has the structure of a coordinate bundle in which the $l(m)$ 's are the coordinate transformations and its topology is the familiar o-topology. The proof of the theorem is therefore reduced to the demonstration that the mapping $h$ is a homeomorphism. We remind the reader that $\pi^{-1}(U)$ has the topology induced by the *-topology in $J(M)$ satisfying conditions (i) $-(\mathrm{v})$.

We first prove that $h$ is continuous. Let $V^{*}$ be an open set in $\pi^{-1}(U)$ and let $\left(m_{0}, v_{0}\right)$ be a point in $V^{*}$. The vector field $X$ for which $X(m)=v_{0}$ for all $m$ in $U$ is continuous on $U$. By (iii) and (v) the associated mapping $\chi$, for which $\chi(m)=\left(m, v_{0}\right)$, is a homeomorphism of $U$ into $\Im(M) \cdot \chi(U) \cap V^{*}$ is open in the topology induced on $\chi(U)$ so $O=\pi\left(\chi(U) \cap V^{*}\right)$ is an open subset of $U$. Since $\left(m_{0}, v_{0}\right) \in \chi(U)$ we have $m_{0} \in O$.

For each $m \in O$ let $W_{m}^{*}=\pi^{-1}(m)-\left(\pi^{-1}(m) \cap V^{*}\right)$ and let $\lambda(m)$ be the infimum of the lengths of all vectors $w-w_{0}$ where $h(m, w) \in W_{m}^{*}$ and $h\left(m, w_{0}\right)=\left(m, v_{0}\right)$. We shall prove that there is a neighborhood $N$ of $m_{0}, N \subset O$, and a constant $\epsilon>0$ such that $\lambda(m) \geqq \epsilon$ for $m \in N$. This will complete the proof of the continuity of $h$ since we may then construct the neighborhood $N \times W$ where

$$
W=\left\{w \mid w \in V^{n} \text {, length of } w-w_{0}<\epsilon\right\} .
$$

$N \times W$ is a neighborhood of $\left(m_{0}, w_{0}\right)$ and $h(N \times W) \subset V^{*}$.

Assume that no such neighborhood $N$ and constant $\epsilon$ exist. Then 
there is a Cauchy sequence $\left(m_{1}, w_{1}\right),\left(m_{2}, w_{2}\right), \cdots$ of points of $O \times V^{n}$ with $h\left(m_{i}, w_{i}\right) \in W_{m_{i}}^{*}$ which converges to $\left(m_{0}, w_{0}\right)$. If we suppose that there are an infinite number of distinct points among $m_{1}, m_{2}, \cdots$ then by passing to a subsequence we may assume that $m_{1}, m_{2}, \cdots$ are themselves distinct. Let $F=\left\{m_{1}, m_{2}, \cdots\right\}$. Then $F \cup\left\{m_{0}\right\}$ is a closed set. The correspondence $m_{i} \rightarrow w_{i}, i=0,1,2, \cdots$, is a section of $U \times V^{n}$ over $F \cup\left\{m_{0}\right\}$. Since $V^{n}$ is a solid space this section over $F \cup\left\{m_{0}\right\}$ may be extended to a section $\phi$ of $U \times V^{n}$ over $U$. The mapping $\chi=h \phi$ of $U$ into $J(M)$ defines a vector field which is continuous on $U$. By (v) the mapping $\chi$ is a homeomorphism of $U$ into $\Im(M)$. Thus $\chi\left(m_{0}\right)$ must be a limit point of $\chi(F)$. But $\chi\left(m_{i}\right)$ $=h \phi\left(m_{i}\right)=h\left(m_{i}, w_{i}\right) \notin V^{*}$ for $i=1,2, \cdots$, and this means that $\chi\left(m_{0}\right)=\left(m_{0}, v_{0}\right)$ is not in $V^{*}$ since $V^{*}$ is open. Since this is not true we conclude that there are only a finite number of points among $m_{1}, m_{2}, \cdots$.

By passing to a subsequence we may assume that $m_{1}=m_{2}=\cdots$ $=m_{0}$ and hence that $h\left(m_{1}, w_{1}\right), h\left(m_{2}, w_{2}\right), \cdots$ are all in $\pi^{-1}\left(m_{0}\right)$. By (iv) the sequence $h\left(m_{0}, w_{1}\right), h\left(m_{0}, w_{2}\right), \cdots$ converges to $h\left(m_{0}, w_{0}\right)$ $=\left(m_{0}, v_{0}\right)$ in $\pi^{-1}\left(m_{0}\right)$. But $h\left(m_{i}, w_{i}\right) \notin V^{*}$ for $i=1,2, \cdots$, so again we find that $\left(m_{0}, v_{0}\right) \notin V^{*}$ which is not true. This completes the proof that $h$ is continuous.

There remains the demonstration that $h^{-1}$ is continuous. Let $W$ be an open set in $U \times V^{n}$, let $\left(m_{0}, w_{0}\right) \in W$, and let $h\left(m_{0}, w_{0}\right)=\left(m_{0}, v_{0}\right)$. Suppose that for every open subset $V^{*}$ of $\pi^{-1}(U)$ which contains $\left(m_{0}, v_{0}\right)$ we find that $h^{-1}\left(V^{*}\right)$ is not contained in $W$. Let

$$
C^{*}=\left\{z^{*} \mid z^{*} \in \pi^{-1}\left(m_{0}\right), z^{*} \notin h(W)\right\} .
$$

Since $h^{-1}\left(C^{*}\right)$ is closed as a subset of the space $(\pi h)^{-1}\left(m_{0}\right)$ so by (iv) $C^{*}$ is closed as a subset of the space $\pi^{-1}\left(m_{0}\right)$. By (iii) $\pi^{-1}\left(m_{0}\right)$ is a closed set so $C^{*}$ is a closed set in $\pi^{-1}(U)$. We shall show that the point $\left(m_{0}, v_{0}\right)$ and the closed set $C^{*}$ cannot be separated by disjoint neighborhoods. This will contradict condition (i) and thereby prove that $h^{-1}$ is continuous.

By (ii) there is an open set $B^{*}$ containing $\left(m_{0}, v_{0}\right)$ such that the closure of $h^{-1}\left(B^{*}\right)$ is compact. Let $V^{*}$ be any open set containing $\left(m_{0}, v_{0}\right)$ and let $N_{1} \supset N_{2} \supset \cdots$ be a nested sequence of open neighborhoods of $m_{0}$ in $M$ such that the diameter of $N_{j} \rightarrow 0$ as $j \rightarrow \infty$. By (iii) $V_{j}^{*}=B^{*} \cap \pi^{-1}\left(N_{j}\right) \cap V^{*}$ is an open set containing $\left(m_{0}, v_{0}\right)$, and by our assumption $h^{-1}\left(V_{j}^{*}\right)$ contains a point $\left(m_{j}, w_{j}\right)$ such that $\left(m_{j}, w_{j}\right)$ $\notin W$. Thus $\left(m_{1}, w_{1}\right),\left(m_{2}, w_{2}\right), \cdots$ is a sequence of points in the compact closure of $h^{-1}\left(B^{*}\right)$ with $m_{j} \rightarrow m_{0}$ as $j \rightarrow \infty$. By passing to a subsequence we may assume that $\left(m_{1}, w_{1}\right),\left(m_{2}, w_{2}\right), \cdots$ is a Cauchy 
sequence. Let $\left(m_{0}, w_{\infty}\right)$ be the limit point. Since $W$ is an open set $\left(m_{0}, w_{\infty}\right) \notin W$. On the other hand, $\left(m_{1}, w_{1}\right),\left(m_{2}, w_{2}\right), \cdots$ are all in $h^{-1}\left(V^{*}\right)$ so that $\left(m_{0}, w_{\infty}\right)$ is in the closure of $h^{-1}\left(V^{*}\right)$. Since $h$ is continuous $h\left(m_{0}, w_{\infty}\right)$ is in the closure of $V^{*}$. We have thus proved that the closure of any open set of $\pi^{-1}(U)$ which contains $\left(m_{0}, v_{0}\right)$ must contain a point of $C^{*}$. It follows that $C^{*}$ and $\left(m_{0}, v_{0}\right)$ cannot be separated by disjoint neighborhoods and the proof of the continuity of $h^{-1}$ is complete.

4. An example. In this section we shall construct an example which illustrates the essential character of condition (ii), for it produces a $*$-topology on $\Im(M)$ which satisfies conditions (iii), (iv), and (v) and is even normal, thereby satisfying condition (i), and yet it fails to satisfy condition (ii) and is therefore not the o-topology.

Let $\mathcal{U}$ be Euclidean 3 -space and let $D$ be the discrete subgroup of $\vartheta$ generated by the vectors $(0,1,0)$ and $(0,0,1)$. The quotient group $\mathfrak{T C}=V / D$ is then a 3-dimensional manifold. Let $\alpha$ be a fixed irrational real number, $0<\alpha<1$. Let $\mathcal{P}$ be the 2 -dimensional subspace of $v$ generated by the vectors $(1,0,0)$ and $(0,1, \alpha)$. Let $J$ be the subset of $\mathfrak{T}$ consisting of those points which are represented by elements of $P$. Since no two distinct points of $\rho$ can represent the same element of $\mathfrak{T}$ the natural mapping of $\mathcal{V}$ onto $\mathfrak{T}$ induces a one-to-one correspondence $h$ between the points of $\odot$ and those of $J . h$ is a continuous mapping of $P$ onto $J$.

Let $M$ be the 1-dimensional subspace of $\rho$ generated by the vector $(1,0,0) . M$ is the real line, a one-dimensional manifold. The point $(x, y, \alpha y)$ of $\rho$ will be denoted simply by $(x, y) . \odot$ has the topology of Euclidean 2-space and is a model for $\Im(M)$ with the o-topology. The point $h(x, y, \alpha y)$ of $J$ will be denoted by $h(x, y)$. The topology induced on $J$ by the topology in $\mathfrak{T}$ will be called the $\dagger$-topology.

If $\left(x_{0}, y_{0}\right) \in \odot$ let

$$
S_{\epsilon}\left(x_{0}, y_{0}\right)=\left\{(x, y, z) \mid\left(x-x_{0}\right)^{2}+\left(y-y_{0}\right)^{2}+\left(z-\alpha y_{0}\right)^{2}<\epsilon^{2}\right\} .
$$

The sets $S_{\epsilon}\left(x_{0}, y_{0}\right) \cap \odot$ are a basis for the o-topology on $\odot$. On the other hand, the sets

$$
U_{\epsilon}\left(x_{0}, y_{0}\right)=h\left(S_{\epsilon}\left(x_{0}, y_{0}\right)\right) \cap \Im
$$

are a basis for the $\dagger$-topology on J. Let

$$
F\left(x_{0}\right)=\left\{\left(x_{0}, y\right) \mid-\infty<y<\infty\right\} .
$$

Let

$$
V_{\epsilon}\left(x_{0}, y_{0}\right)=\left[U_{\epsilon}\left(x_{0}, y_{0}\right)-h\left(F\left(x_{0}\right)\right)\right] \cup h\left[F\left(x_{0}\right) \cap S_{\epsilon}\left(x_{0}, y_{0}\right)\right] .
$$


We take the family of all sets $U_{\epsilon}\left(x_{0}, y_{0}\right)$ and $V_{\epsilon}\left(x_{0}, y_{0}\right)$ as the basis for the *-topology in $J$. Note that $h$ is still a continuous mapping of $\rho$ onto $J$ with this topology.

The projection $\pi$ is defined by $\pi[h(x, y)]=x . \pi$ is continuous in the *-topology so condition (iii) is satisfied. Furthermore, due to the presence of the sets $V_{\epsilon}\left(x_{0}, y_{0}\right)$, the topology induced on $h\left(F\left(x_{0}\right)\right)$ $=\pi^{-1}\left(x_{0}\right)$ by the $*$-topology is the same as that induced on $F\left(x_{0}\right)$ by the o-topology, that is, condition (iv) is satisfied.

Let $X(x)$ be a vector field on $M$. Then $X\left(x_{0}\right)=y_{0}(\partial / \partial x)_{x_{0}}$ for each $x_{0} \in M$ and we define $\phi\left(x_{0}\right)=\left(x_{0}, y_{0}\right) . \phi$ is then a mapping of $M$ into $\odot$. If $X(x)$ is continuous on $M$ then $\phi$ is continuous and hence $\chi=h \phi$ is a continuous mapping of $M$ into $J$. But $\chi$ is the mapping of $M$ into $J$ associated with $X(x)$ and we see that condition (v) is satisfied.

Let $C^{*}$ be a closed set in $J$ and let $p$ be a point of $J$ which is not in $C^{*}$. Then $C^{*}$ is also closed in the $\dagger$-topology. Thus $C^{*}=C \cap J$ where $C$ is a closed subset of the manifold $\mathfrak{T}$ and $p \notin C$. Since $\mathfrak{M}$ is normal we may find disjoint open sets $U$ and $V$ in $\Re$ such that $p \in U$ and $C \subset V$. Let $U^{*}=U \cap J$ and $V^{*}=V \cap J$. Then $U^{*}$ and $V^{*}$ are disjoint open sets in the $\dagger$-topology and hence also in the *-topology. Since $p \in U^{*}$ and $C^{*} \subset V^{*}$ we see that $J$ is normal in the *-topology. On the other hand, each set $V_{\epsilon}\left(x_{0}, y_{0}\right)$ or $U_{\epsilon}\left(x_{0}, y_{0}\right)$ contains points $h(x, y)$ such that $(x, y)$ is arbitrarily distant from $(0,0)$ in the 0 topology. Consequently condition (ii) is violated at each point of $T$.

INDIANA UNIVERSITY 